\title{
Robotic gastrectomy for gastric cancer
}

\author{
Masanori Tokunaga, Masahiro Watanabe, Shizuki Sugita, Akiko Tonouchi, Akio Kaito, Takahiro Kinoshita \\ Gastric Cancer Division, National Cancer Center Hospital East, Kashiwa 277-8577, Japan. \\ Correspondence to: Dr. Masanori Tokunaga, Gastric Cancer Division, National Cancer Center Hospital East, 6-5-1 Kashiwanoha, \\ Kashiwa 277-8577, Japan. E-mail: mtokunag@east.ncc.go.jp
}

\begin{abstract}
How to cite this article: Tokunaga M, Watanabe M, Sugita S, Tonouchi A, Kaito A, Kinoshita T. Robotic gastrectomy for gastric cancer. J Cancer Metastasis Treat2018;4:40. http://dx.doi.org/10.20517/2394-4722.2017.80
\end{abstract}

Received: 21 Nov 2017 First Decision: 23 Jan 2018 Revised: 13 Feb 2018 Accepted: 14 Jul 2018 Published: 30 Jul 2018

Science Editors: Lucio Miele, Masayuki Watanabe Copy Editor: Jun-Yao Li Production Editor: Cai-Hong Wang

\begin{abstract}
Robotic gastrectomy (RG) is increasingly performed, particularly in East Asia. With articulated devices, surgeons are able to perform every procedure more comfortably and meticulously, which makes RG ideal from the surgeon's standpoint. However, it is still unclear whether it is a suitable treatment strategy from the patient's viewpoint, due to the lack of solid evidence obtained from randomized controlled trials. The feasibility of RG has been demonstrated in many retrospective comparative studies, which showed similar trends, including relatively less estimated blood loss and longer operation time with RG than laparoscopic gastrectomy (LG), equivalent number of harvested lymph nodes and similar length of postoperative hospital stay between RG and LG. However, considering the higher medical expenses associated with RG, its superiority in terms of long-term survival outcomes will need to be confirmed for it to be accepted more widely.
\end{abstract}

Keywords: da Vinci, robot, gastric cancer, robot assisted gastrectomy, laparoscopic gastrectomy

\section{INTRODUCTION}

Minimally invasive surgery (MIS) for gastric cancer has been increasingly performed in the East, where incidence of the disease is high and approximately half of cases are diagnosed at an early stage ${ }^{[1-3]}$. The non-inferiority of laparoscopic gastrectomy (LG) for early gastric cancer comparing to open gastrectomy in terms of short- and/or long-term outcomes has been confirmed by randomized controlled trials, and that for advanced gastric cancer is under investigation and may be shown in the near future ${ }^{[4-7]}$. However, LG has several shortcomings which include limitation in the movement range of forceps and the two-dimensional surgical view available to operating surgeons, and it will be necessary to overcome these issues for MIS to be accepted more widely. 

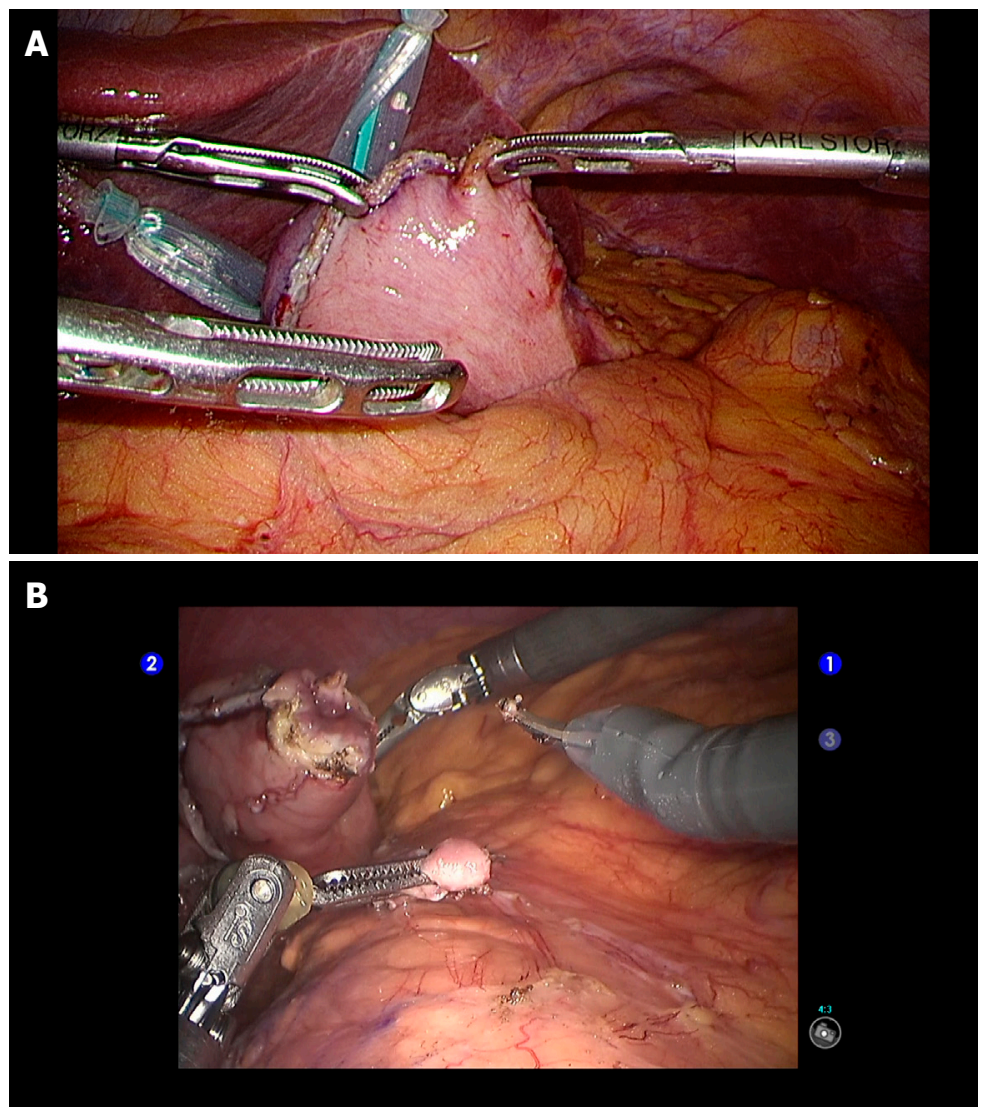

Figure 1. (A) Surgical field during LG: straight devices without articulation are used; (B) surgical field during RG: articulated devices are used

Using the da Vinci ${ }^{\circledR}$ Surgical System (Intuitive Surgical, Sunnyvale, CA, USA), a system for robotic surgery, surgeons are able to attain a three-dimensional view, instrument flexibility, tremor suppression, and improved ergonomics, which are thought to be advantages of robotic gastrectomy $(\mathrm{RG})^{[8-11]}$. With these advantages, theoretically, RG enables surgeons to perform more precise surgery with less trauma, which could result in superior outcomes over LG. However, the number of comparative prospective studies between RG and LG is quite limited, and therefore, solid evidence supporting RG does not yet exist ${ }^{[12-16]}$.

Herein, we would review the comparative retrospective and prospective studies which have investigated the differences in short- and long-term surgical outcomes between RG and LG.

\section{Clear advantages of RG over LG}

There are several clear benefits of RG which contribute to reducing invasiveness and trauma compared with LG. Articulated devices, which are only available in RG, make each surgical technique more meticulous and precise, and are thought to be one definitive advantage of RG [Figure 1 $]^{[8-13]}$. Other apparent advantages include a tremor suppression function, which is helpful to keep a stable surgical field and effective to reduce organ injury, and a three-dimensional image, which has become available in LG although special equipment is necessary. With these clear advantages, RG is expected to have advantages over LG. Clear and possible advantages and disadvantages of both procedures are summarized in Table 1.

\section{Clear disadvantages of RG}

Because RG requires expensive machines and devices, cost effectiveness is an intriguing issue for surgeons, and seems to be an absolute disadvantage of RG. In Korea and Japan, where more than half of reports have 
Table 1. Advantages and disadvantages of RG vs. LG are summarized

\begin{tabular}{ll}
\hline Articulated devices & RG favor \\
\hline 3D image & RG favor \\
Tremor suppression & RG favor \\
ergonomics & RG favor \\
Intraoperative blood loss & Equivalent \\
Morbidity rate & Equivalent \\
Mortality rate & Equivalent \\
Medical expense & LG favor \\
Operation time & LG favor \\
\hline
\end{tabular}

RG: robotic gastrectomy; LG: laparoscopic gastrectomy

been published, the cost for RG is not yet reimbursed by government, and therefore patients or hospitals have to pay additional fees ${ }^{[17]}$. In contrast, medical expense for LG is partially covered by national insurance systems, and the cost burden on patients and hospitals is obviously less than for RG. The additional fee for RG differs between surgeries depending on how many disposable and re-usable instruments are used. Previously, some comparative studies investigated the difference in medical expense between RG and LG and reported that RG expenses were approximately twice as great ${ }^{[18-21]}$. In a prospective comparative study conducted in Korea, significantly higher total cost in the RG group (US\$13,432) than the LG group (US\$8090) was also reported ${ }^{[14]}$. However, if medical expenses associated with RG decrease in the future, they will no longer be an absolute disadvantage of RG.

\section{COMPARISON OF SHORT-TERM SURGICAL OUTCOMES BETWEEN RG AND LG}

Short-term surgical outcomes between RG and LG have been compared in many retrospective and a few prospective studies ${ }^{[9,14-20,22-44]}$. Among short-term surgical outcomes, intraoperative blood loss, the duration of surgery, the number of retrieved lymph nodes, the incidence of postoperative complications, and the length of postoperative hospital stay are thought to reflect surgical quality, and were assessed in most studies.

Intraoperative blood loss was generally equivalent or less during RG than LG [Table 2]. The magnified fine three-dimensional view attained in RG enables surgeons to recognize even very small vessels, and with articulated devices, they can surely stanch bleeding. However, the reported statistically significant differences in intraoperative bleeding between LG and RG were generally less than $100 \mathrm{~mL}$ except for one report from Korea ${ }^{[38]}$, and it is unclear whether the difference is clinically significant of not. Statistically significant more blood loss in RG was also reported in two Japanese studies, but the differences were less than $20 \mathrm{~mL}^{[33,41]}$.

The duration of surgery is significantly longer in RG than in LG in all report, and the difference was statistically significant in most series [Table 3]. Although the difference ranged from 14 to $124 \mathrm{~min}$, it took RG generally approximately 60 min more operation time than LG. There are several probable explanations for longer operation time in RG. Firstly, it takes 15 to $30 \mathrm{~min}$, known as docking time, to prepare before an operator begins the surgery at a console. Secondly, during RG, a surgeon uses four robotic arms, which is less than the average number of five ports used during conventional LG. Although an additional port for an assistant can be used in RG, it is under the assistant's not the surgeon's control, and is sometimes useless due to collisions with robotic arms. As a result, it becomes difficult to make a fine surgical field, particularly in patients with high visceral fat volume or advanced disease, and therefore might cause longer operation time.

The number of retrieved lymph nodes was reported to be almost equal between RG and LG. The duration of postoperative hospital stay was also similar, although a few investigators reported that it was shorter following RG than LG. 
Table 2. Comparison of blood loss

\begin{tabular}{|c|c|c|c|c|c|}
\hline Author & Year & Country/area & Approach & $\begin{array}{c}\text { Number of patients } \\
(n)\end{array}$ & $\begin{array}{c}\text { Blood loss } \\
(\mathrm{mL})\end{array}$ \\
\hline Kimet al. ${ }^{[30]}$ & 2010 & Korea & ODG vs. LDG vs. RDG & 12 vs. 11 vs. 16 & ${ }^{a} 79$ vs. 45 vs. 30 \\
\hline Caruso et al. ${ }^{[22]}$ & 2011 & Italy & OG vs. RG & 120 vs. 29 & 386 vs. 198 \\
\hline Woo et al. ${ }^{[42]}$ & 2011 & Korea & LG vs. RG & 591 vs. 236 & ${ }^{\mathrm{a}} 148$ vs. $92^{* *}$ \\
\hline Huang et $a ._{.[25]}$ & 2012 & Korea & OG vs. LG vs. RG & 586 vs. 64 vs. 39 & ${ }^{\mathrm{a}} 400$ vs. 100 vs. 50 "* \\
\hline Kim et al. ${ }^{[29]}$ & 2012 & Korea & OG vs. LG vs. RG & 4542 vs. 861 vs. 436 & ${ }^{\mathrm{a}} 192$ vs. 112 vs. $85^{* *}$ \\
\hline Uyama et al. ${ }^{[41]}$ & 2012 & Japan & LDG vs. RDG & 25 vs. 225 & ${ }^{\mathrm{a}} 81$ vs. $52^{* *}$ \\
\hline Huang et al. ${ }^{[19]}$ & 2014 & Taiwan & LG vs. RG & 73 vs. 35 & ${ }^{\mathrm{a}} 116 \mathrm{vs} .80^{* *}$ \\
\hline Junfeng et al. ${ }^{[27]}$ & 2014 & America & $L G v s . R G$ & 394 vs. 120 & ${ }^{\mathrm{a}} 138$ vs. $118^{\text {t* }}$ \\
\hline Kimet al. ${ }^{[28]}$ & 2014 & Korea & LDG vs. RDG & 481 vs. 172 & ${ }^{\mathrm{a}} 135$ vs. $60^{* \prime}$ \\
\hline Lee et $a l^{[32]}$ & 2015 & Korea & LDG vs. RDG & 267 vs. 133 & ${ }^{\mathrm{a}} 87 \mathrm{vs} .47^{* *}$ \\
\hline Seo et $a l^{[37]}$ & 2015 & Korea & LDG vs. RDG & 40 vs. 40 & ${ }^{\mathrm{a}} 227$ vs. $76^{\mathrm{**}}$ \\
\hline Suda et al. ${ }^{[40]}$ & 2015 & Japan & LG vs. RG & 438 vs. 88 & ${ }^{\mathrm{a}} 34 \mathrm{vs.} 48^{\circ}$ \\
\hline Nakauchi et al. ${ }^{[17]}$ & 2016 & Japan & $L G v s . R G$ & 437 vs. 84 & ${ }^{\mathrm{a}} 33$ vs. $44^{*}$ \\
\hline Procopiuc et al.[36] & 2016 & Romania & OG vs. RG & 29 vs. 18 & ${ }^{a} 564$ vs. $208^{* *}$ \\
\hline Shen et al. ${ }^{[38]}$ & 2016 & China & LG vs. RG & 330 vs. 93 & ${ }^{\mathrm{a}} 213$ vs. $177^{* *}$ \\
\hline Yang et $a]_{.}^{[43]}$ & 2017 & Korea & OG vs. LG vs. RG & 241 vs. 511 vs. 173 & ${ }^{\mathrm{a}} 149$ vs. 66 vs. $53^{* *}$ \\
\hline \multirow[t]{2}{*}{ Song et al. ${ }^{[9]}$} & 2009 & Korea & LDG (early) vs. RDG & 20 vs. 20 & - \\
\hline & & & LDG (later) vs. RDG & 20 vs. 20 & 40 vs. $94^{* *}$ \\
\hline Eom et al. ${ }^{[18]}$ & 2012 & Korea & LDG vs. RDG & 62 vs. 30 & 88 vs. $153^{* *}$ \\
\hline Park et al. ${ }^{[20]}$ & 2012 & Korea & LDG vs. RDG & 120 vs. 30 & 60 vs. $75^{*}$ \\
\hline Hyun et $a / .^{[26]}$ & 2013 & Korea & LG vs. RG & 83 vs. 38 & 131 vs. $131^{* *}$ \\
\hline Noshiro et al. ${ }^{[33]}$ & 2014 & Japan & LDG vs. RDG & 460 vs. 21 & 115 vs. $96^{* *}$ \\
\hline Son et al. ${ }^{[39]}$ & 2014 & Korea & LTG vs. RTG & 58 vs. 51 & 211 vs. $153^{* *}$ \\
\hline Park et al. ${ }^{[35]}$ & 2015 & Korea & LG vs. RG & 622 vs. 148 & 146 vs. $171^{* *}$ \\
\hline Cianchi et al. ${ }^{[23]}$ & 2016 & Italy & LDG vs. RDG & 41 vs. 30 & 119 vs. $100 "$ \\
\hline Okumura et al. ${ }^{[34]}$ & 2016 & Korea & OG vs. RG & 132 vs. 49 & 157 vs. $85^{* *}$ \\
\hline
\end{tabular}

${ }^{\star}$ median; ${ }^{\star \star}$ mean. ${ }^{a} P$ < 0.05. LDG: laparoscopic distal gastrectomy; LG: laparoscopic gastrectomy; LTG: laparoscopic total gastrectomy; RDG: robotic distal gastrectomy; RG: robotic gastrectomy; RTG: robotic total gastrectomy; ODG: open distal gastrectomy; OG: open astrectomy

The incidence of postoperative complication was compared between the approaches [Table 4]. Many investigators have thought that RG could be safer than LG, because articulated devices, the three-dimensional image, and the tremor suppression function could make recognition of anatomical structures much easier and lymphadenectomy much safer. However, unexpectedly, significantly lower morbidity rate was reported only in two reports, and the difference, even if morbidity rate was lower in RG than LG, was not statistically significant in other reports ${ }^{[3,41]}$. Considering the current status of LG, which is already a well-established safe procedure, it seems to be very difficult to show that RG could further improve the safety. Mortality rate was not statistically significant between RG and LG in any of the studies, and therefore, both RG and LG seem to be safe procedures in terms of postoperative morbidities and mortality.

\section{Long-term outcomes between RG and LG}

The number of reports focusing on long-term survival outcome is quite limited [Table 5]. Three Korean series, which were from a single institute with different study populations, and one Japanese series, reported longterm outcomes with a median follow up period of at least three years ${ }^{[32,33,35,40]}$. In the Korean series, Lee et al ${ }^{[32]}$ focused on patients undergoing D2 distal gastrectomy, Son et al.$^{[39]}$ included patients undergoing spleen-preserving total gastrectomy, and Okumura et al. ${ }^{[34]}$ compared long-term survival outcomes of elderly (70 years old or older) patients between RG and LG. None of these studies showed significant survival differences. The Japanese series by Nakauchi et al. ${ }^{[17]}$ compared three-year overall and recurrence free survival between RG and LG, and reported that no statistically significant difference was found even after stratification by pathological stage. However, the lack of the results of prospective comparative studies focusing on longterm survival makes it difficult to obtain any conclusive result in terms of long-term survival outcomes. 
Table 3. Comparison of operation time

\begin{tabular}{|c|c|c|c|c|c|}
\hline Author & Year & Country/area & Approach & $\begin{array}{c}\text { Number of patients } \\
(n)\end{array}$ & $\begin{array}{c}\text { Operation time } \\
\text { (min) }\end{array}$ \\
\hline \multirow[t]{2}{*}{ Song et $a{ }^{[9]}$} & 2009 & Korea & LDG (early) vs. RDG & 20 vs. 20 & ${ }^{\mathrm{a}} 290$ vs. $203^{* *}$ \\
\hline & & & LDG (later) vs. RDG & 20 vs. 20 & ${ }^{\mathrm{a}} 134$ vs. $203^{\star *}$ \\
\hline Kim et al. ${ }^{[30]}$ & 2010 & Korea & ODG vs. LDG vs. RDG & 12 vs. 11 vs. 16 & ${ }^{\mathrm{a}} 127$ vs. 204 vs. $259^{* *}$ \\
\hline Caruso et al. ${ }^{[22]}$ & 2011 & Italy & OG vs. RG & 120 vs. 29 & a 222 vs. $290^{* *}$ \\
\hline Woo et al. ${ }^{[42]}$ & 2011 & Korea & $\mathrm{LG} v s . \mathrm{RG}$ & 591 vs. 236 & ${ }^{\mathrm{a}} 171$ vs. $220^{* *}$ \\
\hline Eom et al. ${ }^{[18]}$ & 2012 & Korea & LDG vs. RDG & 62 vs. 30 & ${ }^{\mathrm{a}} 189$ vs. $229^{* *}$ \\
\hline Huang et al. ${ }^{[25]}$ & 2012 & Korea & OG vs. LG vs. RG & 586 vs. 64 vs. 39 & ${ }^{\mathrm{a}} 320$ vs. 350 vs. $430^{* *}$ \\
\hline Kimet al. ${ }^{[29]}$ & 2012 & Korea & OG vs. LG vs. RG & 4542 vs. 861 vs. 436 & ${ }^{\mathrm{a}} 158$ vs. 176 vs. $226^{* \star}$ \\
\hline Park et al.. ${ }^{[20]}$ & 2012 & Korea & LDG vs. RDG & 120 vs. 30 & ${ }^{\mathrm{a}} 140$ vs. $218^{*}$ \\
\hline Yoon et al. ${ }^{[44]}$ & 2012 & Korea & LTG vs. RTG & 65 vs. 36 & ${ }^{\mathrm{a}} 210$ vs. $306^{* *}$ \\
\hline Huang et al.. ${ }^{[19]}$ & 2014 & Taiwan & LG vs. RG & 73 vs. 35 & ${ }^{\mathrm{a}} 330$ vs. $358^{* *}$ \\
\hline Junfeng et $a{ }^{[27]}$ & 2014 & America & LG vs. RG & 394 vs. 120 & ${ }^{\mathrm{a}} 221$ vs. $235^{\star \star}$ \\
\hline Kim et $a l^{[28]}$ & 2014 & Korea & LDG vs. RDG & 481 vs. 172 & ${ }^{\mathrm{a}} 167$ vs. $206^{* *}$ \\
\hline Noshiro et al. ${ }^{[33]}$ & 2014 & Japan & LDG vs. RDG & 460 vs. 21 & a 315 vs. $439^{* *}$ \\
\hline Son et al. ${ }^{[39]}$ & 2014 & Korea & LTG vs. RTG & 58 vs. 51 & ${ }^{\mathrm{a}} 210$ vs. $264^{* *}$ \\
\hline Han et al. ${ }^{[24]}$ & 2015 & Korea & LPPG vs. RPPG & 69 vs. 68 & ${ }^{\mathrm{a}} 194$ vs. $258^{* *}$ \\
\hline Lee et $a{ }^{[32]}$ & 2015 & Korea & LDG vs. RDG & 267 vs. 133 & ${ }^{\mathrm{a}} 171$ vs. $218^{* *}$ \\
\hline Park et al. ${ }^{[35]}$ & 2015 & Korea & LG vs. RG & 622 vs. 148 & ${ }^{\mathrm{a}} 189$ vs. $255^{* *}$ \\
\hline Suda et al. ${ }^{[40]}$ & 2015 & Japan & LG vs. RG & 438 vs. 88 & ${ }^{\mathrm{a}} 361$ vs. $381^{*}$ \\
\hline Cianchi et al. ${ }^{[23]}$ & 2016 & Italy & LDG vs. RDG & 41 vs. 30 & ${ }^{\mathrm{a}} 262$ vs. $323^{* *}$ \\
\hline Kimet al. ${ }^{[31]}$ & 2016 & Korea & LDG vs. RDG & 288 vs. 87 & ${ }^{\mathrm{a}} 230$ vs. $248^{* *}$ \\
\hline Nakauchi et al. ${ }^{[17]}$ & 2016 & Japan & LG vs. RG & 437 vs. 84 & a 361 vs. $378^{*}$ \\
\hline Okumura et al. ${ }^{[34]}$ & 2016 & Korea & OG vs. RG & 132 vs. 49 & ${ }^{\mathrm{a}} 174$ vs. $227^{* *}$ \\
\hline Procopiuc et al. ${ }^{[36]}$ & 2016 & Romania & OG vs. RG & 29 vs. 18 & ${ }^{\mathrm{a}} 243$ vs. $320^{* *}$ \\
\hline Shen et al. ${ }^{[38]}$ & 2016 & China & LG vs. RG & 330 vs. 93 & a 226 vs. $257^{* *}$ \\
\hline Yang et al. ${ }^{[43]}$ & 2017 & Korea & OG vs. LG vs. RG & 241 vs. 511 vs. 173 & ${ }^{\mathrm{a}} 193$ vs. 174 vs. $202^{\text {** }}$ \\
\hline Uyama et al. ${ }^{[41]}$ & 2012 & Japan & LDG vs. RDG & 25 vs. 225 & 345 vs. $361^{\star \star}$ \\
\hline Hyun et al. ${ }^{[26]}$ & 2013 & Korea & LG vs. RG & 83 vs. 38 & 220 vs. $234^{* *}$ \\
\hline Seo et $a l_{.[37]}^{[3]}$ & 2015 & Korea & LDG vs. RDG & 40 vs. 40 & 224 vs. $243^{* *}$ \\
\hline
\end{tabular}

*median; **mean. ${ }^{\star} P$ < 0.05. LDG: Iaparoscopic distal gastrectomy; LG: laparoscopic gastrectomy; LTG: laparoscopic total gastrectomy; LPPG: laparoscopic pylorus preservingl gastrectomy; RDG: robotic distal gastrectomy; RG: robotic gastrectomy; RTG: robotic total gastrectomy; RPPG: robotic pylorus preservingl gastrectomy; ODG: open distal gastrectomy; OG: open gastrectomy

Considering the total medical expense of RG, long-term outcomes of RG need to be better than those of LG, and should be confirmed by future prospective trials.

\section{PROSPECTIVE STUDIES}

Although quite a few retrospective studies already exist, the number of prospective studies, particularly that of prospective comparative studies, is extremely limited so far ${ }^{[12-14,16]}$.

Kim et al.$^{[14]}$ reported the results of a prospective non-randomized comparative study. In their study, a total of 423 patients selected either RG or LG after they received a comprehensive explanation of each procedure, and were matched according to surgeon, extent of gastric resection, and sex. Similar early surgical outcomes including morbidity and mortality rate, except for longer operation time in the RG group were reported.

The results of a single-center prospective randomized trial, in which patients were allocated to either open $(n=153)$ or robotic $(n=158)$ gastrectomy groups, were reported by Wang et al ${ }^{[16]}$. Similar complication rates between the groups, and less estimated blood loss, longer duration of surgery, and shorter postoperative hospital stay in the robotic group than the open group were reported. 
Table 4. Compararison of postoperative morbidy and mortality

\begin{tabular}{|c|c|c|c|c|c|c|}
\hline Author & Year & Country/area & Approach & Number of patients $(n)$ & Morbidity rate & Mortality rate \\
\hline Huang et $a l^{[19]}$ & 2014 & Taiwan & LG $v s . R G$ & 73 vs. 35 & ${ }^{\mathrm{a}} 8 \%$ vs. $13 \%$ & $1.4 \%$ vs. $1.4 \%$ \\
\hline Suda et al. ${ }^{[40]}$ & 2015 & Japan & LG vs. RG & 438 vs. 88 & ${ }^{\mathrm{a}} 11 \%$ vs. $2 \%$ & $0.2 \%$ vs. $1.1 \%$ \\
\hline Nakauchi et al. ${ }^{[17]}$ & 2016 & Japan & LG vs. RG & 437 vs. 84 & ${ }^{\mathrm{a}} 12 \%$ vs. $2 \%$ & - \\
\hline Yang et al. ${ }^{[43]}$ & 2017 & Korea & OG vs. LG vs. RG & 241 vs. 511 vs. 173 & ${ }^{\mathrm{a}} 25 \%$ vs. $12 \%$ vs. $5 \%$ & $0.8 \%$ vs. $0.4 \%$ vs. $0 \%$ \\
\hline \multirow[t]{2}{*}{ Song et al. ${ }^{[9]}$} & 2009 & Korea & LDG (early) vs. RDG & 20 vs. 20 & $5 \%$ vs. $5 \%$ & $0 \%$ vs. $0 \%$ \\
\hline & & & LDG (later) vs. RDG & 20 vs. 20 & $10 \%$ vs. $5 \%$ & $0 \%$ vs. $0 \%$ \\
\hline Kimet al. ${ }^{[30]}$ & 2010 & Korea & ODG vs. LDG vs. RDG & 12 vs. 11 vs. 16 & $17 \%$ vs. $9 \%$ vs. $13 \%$ & $0 \%$ vs. $0 \%$ vs. $0 \%$ \\
\hline Caruso et al. ${ }^{[22]}$ & 2011 & Italy & OG vs. RG & 120 vs. 29 & $43 \%$ vs. $41 \%$ & $3.3 \%$ vs. $0 \%$ \\
\hline Woo et al. ${ }^{[42]}$ & 2011 & Korea & LG vs. RG & 591 vs. 236 & $14 \%$ vs. $11 \%$ & $0.3 \%$ vs. $0.4 \%$ \\
\hline Eom et al. ${ }^{[18]}$ & 2012 & Korea & LDG vs. RDG & 62 vs. 30 & $7 \%$ vs. $13 \%$ & $0 \%$ vs. $0 \%$ \\
\hline Huang et al. ${ }^{[25]}$ & 2012 & Korea & OG vs. LG vs. RG & 586 vs. 64 vs. 39 & $15 \%$ vs. $16 \%$ vs. $15 \%$ & $1.4 \%$ vs. $1.6 \%$ vs. $2.6 \%$ \\
\hline Kim et al. ${ }^{[29]}$ & 2012 & Korea & OG vs. LG vs. RG & 4542 vs. 861 vs. 436 & $11 \%$ vs. $9 \%$ vs. $10 \%$ & $0.5 \%$ vs. $0.3 \%$ vs. $0.5 \%$ \\
\hline Park et al..$^{[20]}$ & 2012 & Korea & LDG vs. RDG & 120 vs. 30 & $8 \%$ vs. $17 \%$ & $0 \%$ vs. $0 \%$ \\
\hline Uyama et al. ${ }^{[41]}$ & 2012 & Japan & LDG vs. RDG & 25 vs. 225 & $11 \%$ vs. $17 \%$ & $0 \%$ vs. 0\% \\
\hline Yoon et al. ${ }^{[44]}$ & 2012 & Korea & LTG vs. RTG & 65 vs. 36 & $15 \%$ vs. $17 \%$ & $0 \%$ vs. $0 \%$ \\
\hline Hyun et al. ${ }^{[26]}$ & 2013 & Korea & LG vs. RG & 83 vs. 38 & $39 \%$ vs. $47 \%$ & $0 \%$ vs. $0 \%$ \\
\hline Junfeng et al. ${ }^{[27]}$ & 2014 & America & LG vs. RG & 394 vs. 120 & $4 \%$ vs. $6 \%$ & - \\
\hline Kim et al. ${ }^{[28]}$ & 2014 & Korea & LDG vs. RDG & 481 vs. 172 & $4 \%$ vs. $5 \%$ & $0.6 \%$ vs. $0 \%$ \\
\hline Noshiro et al. ${ }^{[33]}$ & 2014 & Japan & LDG vs. RDG & 460 vs. 21 & $10 \%$ vs. $10 \%$ & $0 \%$ vs. 0\% \\
\hline Son et al. ${ }^{[39]}$ & 2014 & Korea & LTG vs. RTG & 58 vs. 51 & $22 \%$ vs. $16 \%$ & $0 \%$ vs. $2.0 \%$ \\
\hline Han et al. ${ }^{[24]}$ & 2015 & Korea & LPPG vs. RPPG & 69 vs. 68 & $22 \%$ vs. $19 \%$ & $0 \%$ vs. $0 \%$ \\
\hline Lee et $a l^{[32]}$ & 2015 & Korea & LDG $v s$. RDG & 267 vs. 133 & $13 \%$ vs. $11 \%$ & - \\
\hline Seo et al. ${ }^{[37]}$ & 2015 & Korea & LDG vs. RDG & 40 vs. 40 & $30 \%$ vs. $28 \%$ & - \\
\hline Park et al. ${ }^{[35]}$ & 2015 & Korea & LG vs. RG & 622 vs. 148 & $8 \%$ vs. $8 \%$ & $0.5 \%$ vs. $0 \%$ \\
\hline Cianchi et al. ${ }^{[23]}$ & 2016 & Italy & LDG vs. RDG & 41 vs. 30 & $12 \%$ vs. $13 \%$ & $4.9 \%$ vs. $3.3 \%$ \\
\hline Kim et al. ${ }^{[31]}$ & 2016 & Korea & LDG vs. RDG & 288 vs. 87 & $9 \%$ vs. $6 \%$ & $0.3 \%$ vs. $1.1 \%$ \\
\hline Okumura et al. ${ }^{[34]}$ & 2016 & Korea & OG vs. RG & 132 vs. 49 & $18 \%$ vs. $14 \%$ & 0\% vs. $0 \%$ \\
\hline Procopiuc et al. ${ }^{[36]}$ & 2016 & Romania & OG vs. RG & 29 vs. 18 & $28 \%$ vs. $22 \%$ & $0 \%$ vs. $0 \%$ \\
\hline Shen et al. ${ }^{[38]}$ & 2016 & China & LG vs. RG & 330 vs. 93 & $10 \%$ vs. $10 \%$ & - \\
\hline
\end{tabular}

${ }^{a} P$ < 0.05. LDG: laparoscopic distal gastrectomy; LG: laparoscopic gastrectomy; LTG: laparoscopic total gastrectomy; LPPG: laparoscopic pylorus preservingl gastrectomy; RDG: robotic distal gastrectomy; RG: robotic gastrectomy; RTG: robotic total gastrectomy; RPPG: robotic pylorus preservingl gastrectomy; ODG: open distal gastrectomy; OG: open gastrectomy

Table 5. Studies which provided long-term survival outcomes

\begin{tabular}{|c|c|c|c|c|c|c|c|}
\hline Author & Year & $\begin{array}{c}\text { Country/ } \\
\text { area }\end{array}$ & Approach & $\begin{array}{l}\text { Number of } \\
\text { patients } \\
\text { (n) }\end{array}$ & $\begin{array}{l}\text { Median Follow } \\
\text { up period } \\
\text { (months) }\end{array}$ & $\begin{array}{c}5 y-0 S \\
(\%)\end{array}$ & $\begin{array}{c}\text { 5y-DFS } \\
(\%)\end{array}$ \\
\hline Son et al. ${ }^{[39]}$ & 2014 & Korea & LTG vs. RTG & 58 vs. 51 & ${ }^{a} 70$ & ${ }^{\mathrm{a}} 91.1 \mathrm{vs} .89 .5$ & ${ }^{\mathrm{a}} 90.2$ vs. 91.2 \\
\hline Lee et al. ${ }^{[32]}$ & 2015 & Korea & LDG vs. RDG & 267 vs. 133 & ${ }^{a} 75$ & ${ }^{\mathrm{a} N} . \mathrm{S}$. & - \\
\hline Okumura et al.. ${ }^{[34]}$ & 2016 & Korea & OG vs. RG & 132 vs. 49 & ${ }^{a} 58$ & ${ }^{\mathrm{a}} \mathrm{N} . \mathrm{S}$. & - \\
\hline Junfeng et $a l^{[27]}$ & 2014 & America & LG vs. RG & 394 vs. 120 & 19 vs. 15 & 69.9 vs. 67.8 (3y) & - \\
\hline Han et al. ${ }^{[24]}$ & 2015 & Korea & LPPG vs. RPPG & 69 vs. 68 & 19 vs. 23 & - & - \\
\hline Nakauchi et al. ${ }^{[17]}$ & 2016 & Japan & LG vs. RG & 437 vs. 84 & 42 vs. 41 & 88.8 vs. 86.9 (3y) & 86.3 vs. 86.9 (3y) \\
\hline Procopiuc et al.. ${ }^{[36]}$ & 2016 & Romania & OG vs. RG & 29 vs. 18 & 32 vs. 25 & N.S. & - \\
\hline
\end{tabular}

amedian follow up period longer than 3 years. N.S.: statistically not significant difference; LDG: laparoscopic distal gastrectomy; LG: laparoscopic gastrectomy; LTG: laparoscopic total gastrectomy; LPPG: laparoscopic pylorus preservingl gastrectomy; RDG: robotic distal gastrectomy; RG: robotic gastrectomy; RTG: robotic total gastrectomy; RPPG: robotic pylorus preservingl gastrectomy; OG: open gastrectomy

\section{DISCUSSION}

RG has several absolute advantages, which include articulated devices, tremor suppression function, and a fine three-dimensional view, and surgeons can perform operations comfortably with these technologies. However, these advantages are from the surgeons' perspective, and it is unclear whether these technologies applied to RG are also advantageous from the patients' viewpoint. Theoretically, the more meticulous and precise surgeries are, the better the outcomes will be. However, for RG to be more widely accepted, advantages from the patients' side should be demonstrated in clinical trials, ideally in prospective randomized trials. 
Short-term surgical outcomes such as intraoperative bleeding, surgical time, duration of postoperative hospital stay, and postoperative morbidity and mortality rate are thought to reflect surgical quality, and some of them directly affect patients' quality of life. Therefore, these factors are frequently compared between surgical procedures, when investigators need to show superiority or non-inferiority of a newly emergent procedure. Indeed, they have been compared in many studies of RG and LG. However, it seems difficult to conclude that RG is a superior procedure to LG in terms of short-term surgical outcomes, because RG is a more time-consuming procedure, but does not show any obvious benefits. Although some have reported that RG is associated with less bleeding, the differences, which were generally less than $100 \mathrm{~mL}$, seem not to be clinically meaningful. It might be difficult to demonstrate that RG could further improve short-term surgical outcomes, because LG is already a well-established and satisfactorily safe procedure.

The number of studies focusing on long-term surgical outcomes is quite limited, due to insufficient follow-up period in each study. So far, similar long-term survival outcomes between RG and LG have been reported, and we need to wait for the results of currently ongoing studies to reach any conclusions about long-term survival outcomes.

Interpretation of the results of comparative studies should be done carefully because of possible selection bias. In most comparative studies, surgical approaches were selected by the patients themselves after thoughtful explanation of both procedures, but the possibility of selection bias should be taken into account. To overcome this issue, well designed prospective, hopefully randomized controlled, trials are necessary, and we have to at least wait for the results of prospective non-randomized comparative studies ${ }^{[14]}$.

To demonstrate the feasibility of RG, the surgical outcomes of RG are usually compared with those of LG. However, considering that both surgeries were developed on the concept of being minimally invasive, the differences between RG and LG might be marginal, even if RG is truly a superior procedure to LG. In addition RG is, so far, obviously the more expensive surgical procedure. Therefore, it seems unrealistic for RG to completely replace LG with all surgeries in the very near future. However, if the cost of RG decreases dramatically and high medical expense is no longer a problem, it may be a different story with RG becoming further widespread.

So far, RG seems to be as feasible as LG in terms of short- and long-term surgical outcomes. However, RG is an expensive procedure at present, and it is unclear whether RG is superior to LG from the patients' standpoint. The results of well designed prospective comparative studies are awaited to obtain conclusive results on this issue.

\section{DECLARATIONS}

\section{Authors' contributions}

Analysed and interpreted the data: Tokunaga M, Watanabe M, Sugita S, Tonouchi A, Kaito A, Kinoshita T Read and approved the final manuscript: Tokunaga M, Watanabe M, Sugita S, Tonouchi A, Kaito A, Kinoshita T

\section{Availability of data and meterials \\ Not applicable.}

\section{Financial support and sponsorship}

None.

\section{Conflicts of interest}

All authors declared that there are no conflicts of interest. 


\section{Ethical approval and consent to participate}

Not applicable.

\section{Consent for publication}

Not applicable.

\section{Copyright}

(c) The Author(s) 2018.

\section{REFERENCES}

1. Ohtani H, Tamamori Y, Noguchi K, Azuma T, Fujimoto S, Oba H, Aoki T, Minami M, Hirakawa K. A meta-analysis of randomized controlled trials that compared laparoscopy-assisted and open distal gastrectomy for early gastric cancer. J Gastrointest Surg 2010;14:958-64.

2. Kim YW, Baik YH, Yun YH, Nam BH, Kim DH, Choi IJ, Bae JM. Improved quality of life outcomes after laparoscopy-assisted distal gastrectomy for early gastric cancer: results of a prospective randomized clinical trial. Ann Surg 2008;248:721-7.

3. Kodera Y, Fujiwara M, Ohashi N, Nakayama G, Koike M, Morita S, Nakao A. Laparoscopic surgery for gastric cancer: a collective review with meta-analysis of randomized trials. J Am Coll Surg 2010;211:677-86.

4. Takagi M, Katai H, Mizusawa J, Nakamura K, Yoshikawa T, Terashima M. A phase III study of laparoscopy-assisted versus open distal gastrectomy with nodal dissection for clinical stage IA/IB gastric cancer (JCOG0912): analysis of the sfety and short-term clinical outcomes. J Clin Oncol 2015;33:(suppl: abstr 4017).

5. Kim W, Kim HH, Han SU, Kim MC, Hyung WJ, Ryu SW, Cho GS, Kim CY, Yang HK, Park DJ, Song KY, Lee SI, Ryu SY, Lee JH, Lee HJ; Korean Laparo-endoscopic Gastrointestinal Surgery Study (KLASS) Group. Decreased morbidity of laparoscopic distal gastrectomy compared with open distal gastrectomy for stage i gastric cancer: short-term outcomes from a multicenter randomized controlled trial (KLASS-01). Ann Surg 2016;263:28-35.

6. Kim HH, Han SU, Kim MC, Kim W, Lee HJ, Ryu SW, Cho GS, Kim CY, Yang HK, Park DJ, Song KY, Lee SI, Ryu SY, Lee JH, Hyung WJ. Long term outcomes of laparoscopic distal gastrectomy compared with open distal gastrectomy for clinical stage I gastric adenocarcinoma (KLASS-01): a multi-center prospective randomized controlled trial. J Clin Oncol 2016;34:(suppl: abstr 4060).

7. Hu Y, Huang C, Sun Y, Su X, Cao H, Hu J, Xue Y, Suo J, Tao K, He X, Wei H, Ying M, Hu W, Du X, Chen P, Liu H, Zheng C, Liu F, Yu J, Li Z, Zhao G, Chen X, Wang K, Li P, Xing J, Li G. Morbidity and mortality of laparoscopic versus open D2 distal gastrectomy for advanced gastric cancer: a randomized controlled trial. J Clin Oncol 2016;34:1350-7.

8. Song J, Oh SJ, Kang WH, Hyung WJ, Choi SH, Noh SH. Robot-assisted gastrectomy with lymph node dissection for gastric cancer: lessons learned from an initial 100 consecutive procedures. Ann Surg 2009;249:927-32.

9. Song J, Kang WH, Oh SJ, Hyung WJ, Choi SH, Noh SH. Role of robotic gastrectomy using da Vinci system compared with laparoscopic gastrectomy: initial experience of 20 consecutive cases. Surg Endosc 2009;23:1204-11.

10. Hashizume M, Sugimachi K. Robot-assisted gastric surgery. Surg Clin North Am 2003;83:1429-44.

11. Kakeji Y, Konishi K, Ieiri S, Yasunaga T, Nakamoto M, Tanoue K, Baba H, Maehara Y, Hashizume M. Robotic laparoscopic distal gastrectomy: a comparison of the da Vinci and Zeus systems. Int J Med Robot 2006;2:299-304.

12. Tokunaga M, Makuuchi R, Miki Y, Tanizawa Y, Bando E, Kawamura T, Terashima M. Late phase II study of robot-assisted gastrectomy with nodal dissection for clinical stage I gastric cancer. Surg Endosc 2016;30:3362-7.

13. Tokunaga M, Sugisawa N, Kondo J, Tanizawa Y, Bando E, Kawamura T, Terashima M. Early phase II study of robot-assisted distal gastrectomy with nodal dissection for clinical stage IA gastric cancer. Gastric Cancer 2014;17:542-7.

14. Kim HI, Han SU, Yang HK, Kim YW, Lee HJ, Ryu KW, Park JM, An JY, Kim MC, Park S, Song KY, Oh SJ, Kong SH, Suh BJ, Yang DH, Ha TK, Kim YN, Hyung WJ. Multicenter prospective comparative study of robotic versus laparoscopic gastrectomy for gastric adenocarcinoma. Ann Surg 2016;263:103-9.

15. Park JM, Kim HI, Han SU, Yang HK, Kim YW, Lee HJ, An JY, Kim MC, Park S, Song KY, Oh SJ, Kong SH, Suh BJ, Yang DH, Ha TK, Hyung WJ, Ryu KW. Who may benefit from robotic gastrectomy?: a subgroup analysis of multicenter prospective comparative study data on robotic versus laparoscopic gastrectomy. Eur J Surg Oncol 2016;42:1944-9.

16. Wang G, Jiang Z, Zhao J, Liu J, Zhang S, Zhao K, Feng X, Li J. Assessing the safety and efficacy of full robotic gastrectomy with intracorporeal robot-sewn anastomosis for gastric cancer: a randomized clinical trial. J Surg Oncol 2016;113:397-404.

17. Nakauchi M, Suda K, Susumu S, Kadoya S, Inaba K, Ishida Y, Uyama I. Comparison of the long-term outcomes of robotic radical gastrectomy for gastric cancer and conventional laparoscopic approach: a single institutional retrospective cohort study. Surg Endosc 2016;30:5444-52.

18. Eom BW, Yoon HM, Ryu KW, Lee JH, Cho SJ, Lee JY, Kim CG, Choi IJ, Lee JS, Kook MC, Rhee JY, Park SR, Kim YW. Comparison of surgical performance and short-term clinical outcomes between laparoscopic and robotic surgery in distal gastric cancer. Eur J Surg Oncol 2012;38:57-63.

19. Huang KH, Lan YT, Fang WL, Chen JH, Lo SS, Li AF, Chiou SH, Wu CW, Shyr YM. Comparison of the operative outcomes and learning curves between laparoscopic and robotic gastrectomy for gastric cancer. PLoS One 2014;9:e111499. 
20. Park JY, Jo MJ, Nam BH, Kim Y, Eom BW, Yoon HM, Ryu KW, Kim YW, Lee JH. Surgical stress after robot-assisted distal gastrectomy and its economic implications. Br J Surg 2012;99:1554-61.

21. Greenleaf EK, Sun SX, Hollenbeak CS, Wong J. Minimally invasive surgery for gastric cancer: the American experience. Gastric Cancer 2017;20:368-78.

22. Caruso S, Patriti A, Marrelli D, Ceccarelli G, Ceribelli C, Roviello F, Casciola L. Open vs robot-assisted laparoscopic gastric resection with D2 lymph node dissection for adenocarcinoma: a case-control study. Int J Med Robot 2011;7:452-8.

23. Cianchi F, Indennitate G, Trallori G, Ortolani M, Paoli B, Macri G, Macrì G, Lami G, Mallardi B, Badii B, Staderini F, Qirici E, Taddei A, Ringressi MN, Messerini L, Novelli L, Bagnoli S, Bonanomi A, Foppa C, Skalamera I, Fiorenza G, Perigli G. Robotic vs laparoscopic distal gastrectomy with D2 lymphadenectomy for gastric cancer: a retrospective comparative mono-institutional study. BMC Surg 2016;16:65.

24. Han DS, Suh YS, Ahn HS, Kong SH, Lee HJ, Kim WH, Yang HK. Comparison of surgical outcomes of robot-assisted and laparoscopyassisted pylorus-preserving gastrectomy for gastric cancer: a propensity score matching analysis. Ann Surg Oncol 2015;22:2323-8.

25. Huang KH, Lan YT, Fang WL, Chen JH, Lo SS, Hsieh MC, Li AF, Chiou SH, Wu CW. Initial experience of robotic gastrectomy and comparison with open and laparoscopic gastrectomy for gastric cancer. J Gastrointest Surg 2012;16:1303-10.

26. Hyun MH, Lee CH, Kwon YJ, Cho SI, Jang YJ, Kim DH, Kim JH, Park SH, Mok YJ, Park SS. Robot versus laparoscopic gastrectomy for cancer by an experienced surgeon: comparisons of surgery, complications, and surgical stress. Ann Surg Oncol 2013;20:1258-65.

27. Junfeng Z, Yan S, Bo T, Yingxue H, Dongzhu Z, Yongliang Z, Feng Q, Peiwu Y. Robotic gastrectomy versus laparoscopic gastrectomy for gastric cancer: comparison of surgical performance and short-term outcomes. Surg Endosc 2014;28:1779-87.

28. Kim HI, Park MS, Song KJ, Woo Y, Hyung WJ. Rapid and safe learning of robotic gastrectomy for gastric cancer: multidimensional analysis in a comparison with laparoscopic gastrectomy. Eur J Surg Oncol 2014;40:1346-54.

29. Kim KM, An JY, Kim HI, Cheong JH, Hyung WJ, Noh SH. Major early complications following open, laparoscopic and robotic gastrectomy. Br J Surg 2012;99:1681-7.

30. Kim MC, Heo GU, Jung GJ. Robotic gastrectomy for gastric cancer: surgical techniques and clinical merits. Surg Endosc 2010;24:610-5.

31. Kim YW, Reim D, Park JY, Eom BW, Kook MC, Ryu KW, Yoon HM. Role of robot-assisted distal gastrectomy compared to laparoscopy-assisted distal gastrectomy in suprapancreatic nodal dissection for gastric cancer. Surg Endosc 2016;30:1547-52.

32. Lee J, Kim YM, Woo Y, Obama K, Noh SH, Hyung WJ. Robotic distal subtotal gastrectomy with D2 lymphadenectomy for gastric cancer patients with high body mass index: comparison with conventional laparoscopic distal subtotal gastrectomy with D2 lymphadenectomy. Surg Endosc 2015;29:3251-60.

33. Noshiro H, Ikeda O, Urata M. Robotically-enhanced surgical anatomy enables surgeons to perform distal gastrectomy for gastric cancer using electric cautery devices alone. Surg Endosc 2014;28:1180-7.

34. Okumura N, Son T, Kim YM, Kim HI, An JY, Noh SH, Hyung WJ. Robotic gastrectomy for elderly gastric cancer patients: comparisons with robotic gastrectomy in younger patients and laparoscopic gastrectomy in the elderly. Gastric Cancer 2016;19:1125-34.

35. Park JY, Ryu KW, Reim D, Eom BW, Yoon HM, Rho JY, Choi IJ, Kim YW. Robot-assisted gastrectomy for early gastric cancer: is it beneficial in viscerally obese patients compared to laparoscopic gastrectomy? World J Surg 2015;39:1789-97.

36. Procopiuc L, Tudor S, Manuc M, Diculescu M, Vasilescu C. Open vs robotic radical gastrectomy for locally advanced gastric cancer. Int J Med Robot 2016;12:502-8.

37. Seo HS, Shim JH, Jeon HM, Park CH, Song KY. Postoperative pancreatic fistula after robot distal gastrectomy. J Surg Res 2015;194:361-6.

38. Shen W, Xi H, Wei B, Cui J, Bian S, Zhang K, Wang N, Huang X, Chen L. Robotic versus laparoscopic gastrectomy for gastric cancer: comparison of short-term surgical outcomes. Surg Endosc 2016;30:574-80.

39. Son T, Lee JH, Kim YM, Kim HI, Noh SH, Hyung WJ. Robotic spleen-preserving total gastrectomy for gastric cancer: comparison with conventional laparoscopic procedure. Surg Endosc 2014;28:2606-15.

40. Suda K, Man IM, Ishida Y, Kawamura Y, Satoh S, Uyama I. Potential advantages of robotic radical gastrectomy for gastric adenocarcinoma in comparison with conventional laparoscopic approach: a single institutional retrospective comparative cohort study. Surg Endosc 2015;29:673-85.

41. Uyama I, Kanaya S, Ishida Y, Inaba K, Suda K, Satoh S. Novel integrated robotic approach for suprapancreatic D2 nodal dissection for treating gastric cancer: technique and initial experience. World J Surg 2012;36:331-7.

42. Woo Y, Hyung WJ, Pak KH, Inaba K, Obama K, Choi SH, Noh SH. Robotic gastrectomy as an oncologically sound alternative to laparoscopic resections for the treatment of early-stage gastric cancers. Arch Surg 2011;146:1086-92.

43. Yang SY, Roh KH, Kim YN, Cho M, Lim SH, Son T, Hyung WJ, Kim HI. Surgical outcomes after open, laparoscopic, and robotic gastrectomy for gastric cancer. Ann Surg Oncol 2017;24:1770-7.

44. Yoon HM, Kim YW, Lee JH, Ryu KW, Eom BW, Park JY, Choi IJ, Kim CG, Lee JY, Cho SJ, Rho JY. Robot-assisted total gastrectomy is comparable with laparoscopically assisted total gastrectomy for early gastric cancer. Surg Endosc 2012;26:1377-81. 